\title{
Identification of focal epileptic regions from electroencephalographic data: Feigenbaum graphs
}

\author{
G. Guarneros ${ }^{a}$, C. Pérez ${ }^{a}$, A. Montiel ${ }^{a}$ and J. F. Rojas ${ }^{a, b}$ \\ ${ }^{a}$ Facultad de Ciencias Físico Matemáticas. Benemérita Universidad Autónoma de Puebla, \\ Av. San Claudio y 18 sur, Ciudad Universitaria, Col. San Manuel, 72570. Puebla, México. \\ ${ }^{b}$ Centro Multidisciplinario de Modelación Matemática y Computacional, Benemérita Universidad Autónoma de Puebla, \\ Av. San Claudio y 18 sur, Ciudad Universitaria, Col. San Manuel, 72570. Puebla, México.
}

Received 30 August 2020; accepted 29 May 2020

In the study of problems related to epilepsy, analyzing electroencephalographic data is of much importance help to, e.g., diagnose and to diminish errors in surgery. In this work, we present an analysis via the construction of Feigenbaum graphs by using real electroencephalographic signals data sets and calculating characteristic network (graph) quantities, such as average clustering, degree distribution, and average shortest path length. By using this method, we manage to characterize two different data sets from each other, from data sets corresponding to focal and non-focal neuronal activity both recorded out of an epileptic seizure. This method makes it possible to identify sets of data from epileptic focal zones, and we suggest that this approach could be used to aid physicians in diagnosing epilepsy from electroencephalographic data and in the exact establishment of the epileptic focal region for surgery.

Keywords: EEG; epilepsy; statistical physics methods; Feigenbaum graphs; visibility graph.

PACS: 02.90.+p; 02.70.-c; 05.45.Tp; 87.15.A-; 87.19.le

DOI: https://doi.org/10.31349/RevMexFis.67.324

\section{Introduction}

Epilepsy is a disease that affects sixty-five million people in different countries, and two and a half million new cases are detected every year [1]. Epilepsy is a disease characterized by an enduring predisposition to generate epileptic seizures and the neurobiological, cognitive, psychological, and social consequences of this condition [2].

Epileptic people are two or three times more likely to die prematurely [3]. $50 \%$ of the cases begin in childhood or adolescence [4]. Epilepsy is characterized by seizures, which can affect persons of any age. The seizures can be as sparse as once a year or as often as several times a day [5]. Given these factors, the importance of diagnosing epilepsy is very high [6], so the tools and techniques used and developed for this end are too [7-11].

Seizure disorders are not epileptic in nature; or, in other words, not all seizures are epileptic fits. Epileptic seizures are unprovoked due to the involvement of the central nervous system. Non-epileptic seizures could be due to several measurable causes, such as stroke, dementia, head injury, brain infections, congenital birth defects, birth-related brain injuries, tumors, and other space-occupying lesions $[12,13]$.

One of the procedures for diagnosing epilepsy consists of the analysis of electroencephalographic signals (EEG) of a patient [14]. The EEG measures the electrical activity of the cortical area by means of electrodes placed on the scalp of the patient [15]. More accurately, it measures the electrical potential of the dendrites of the pyramidal neurons adjacent to the cortical surface. Hence, the relevance of EEG analysis in diagnosing neural disorders, and epilepsy in particular [16].
Since EEG recordings are, in essence, a time series with lots of noise $[17,18]$, the task of analyzing and achieving a diagnosis becomes a very difficult one $[19,20]$. Because it is such a difficult procedure, it requires a very well trained physician [6]. That is why many scientists are trying to develop techniques to ease this workload and facilitate the physician's job [21-23].

One field of study of much relevance is the automated EEG analysis, which includes many computer-aided algorithms, such as component analysis [24], Fourier Transform $[25,26]$, wavelet transform $[27,28]$, and entropy analysis [29$31]$ among others $[23,25,30,32,33]$.

Zhong-Ke Gao et al. [34] used a hybrid method of making a visual graph out of an adaptive optimal kernel timefrequency representation of the EEG. They manage to detect epileptic seizures from EEG data by means of statistical measurements of the visual graph, such as clustering coefficient and clustering entropy.

Salim Lahmiri [35] made a statistical analysis of EEG signals by measuring the Generalised Hurst exponent. He shows statistical differences between the estimated Generalised Hurst exponent for normal EEG signals and EEG signals with epileptic activity.

Lei Wang et al. [36] used visibility graphs to analyse seizure patterns in EEG signals. By calculating and comparing degree distributions they manage to show that it can be used to discern between EEG recording with and without seizures.

A new method to characterize EEG signals is proposed. This method can be used to identify epileptic regions by means of the associated Feigenbaum graphs. By turning the 


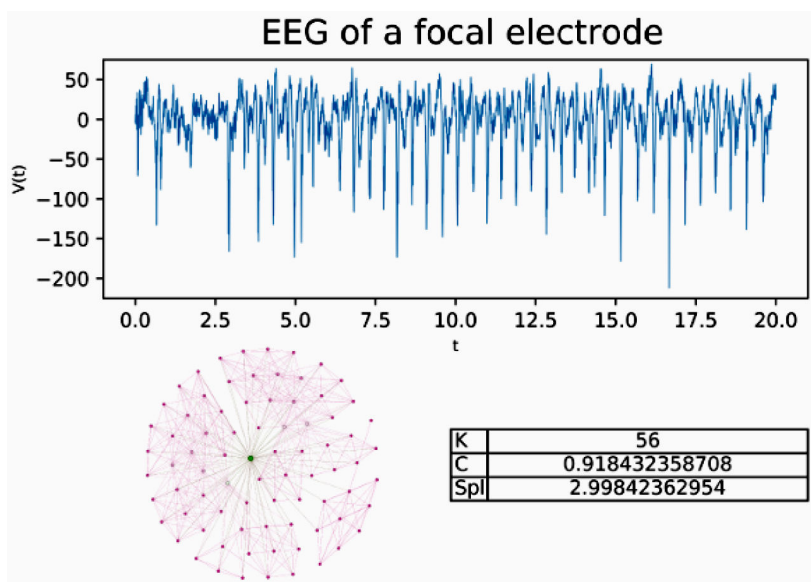

(b)

(c)

FIGURE 1. 20 seconds of EEG activity from a focal electrode. b) shows a subnetwork for the EEG built by Feigenbaum approach, c) shows the values for average degree $(K)$, average clustering $(C)$ and average shortest path length $(S P L)$.

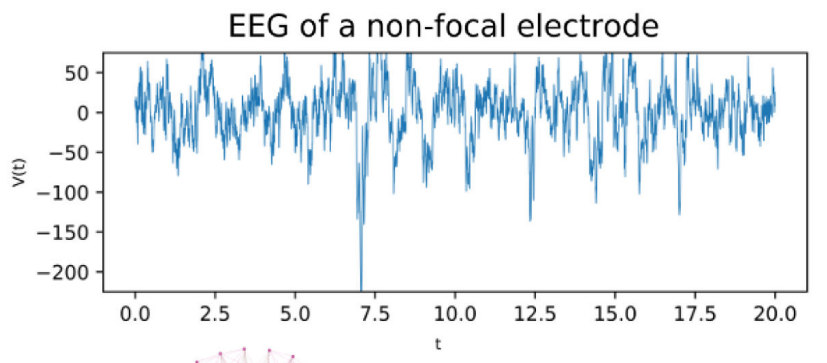

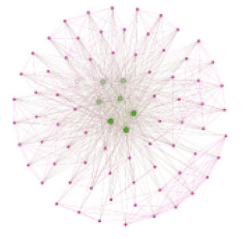

(b)

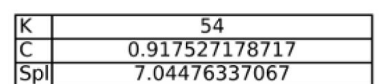

(c)
FIGURE 2. 20 seconds of EEG activity from a non-focal electrode. b) shows a subnetwork for the EEG built by Feigenbaum approach, c) shows the values for average degree $(k)$, average clustering $(C)$ and average shortest path length $(S P L)$.

EEG time series into graphs, they can be studied through their topology. This is made by calculating and statistically checking the average clustering coefficient and the average shortest path length of the graphs.

In the construction of the Feigenbaum graph from the data, most of the detailed information of the time series will be reflected in some properties of the obtained network topology and its statistical measurements. In this study, the Feigenbaum graphs are used to analyze EEG data from the Andrzejak et al. study [37]. Using statistical criteria, average shortest path length, and average clustering coefficient were used to discern between signals " $F$ " from a focal region and signals " $N$ " from the non-focal region.

In Figs. 1 and 2, the Feigenbaum network is shown, along with the values of the parameters, average degree $(K)$, average clustering $(C)$, and average shortest path length $(S P L)$ for two samples of 20 seconds type "F" and type "N" respectively.

\section{EEG signals}

The Data used was taken from the publicly available source of the Bern-Barcelona EEG database [37], where Andrzejak et al. originally made a correlation study.

The data were taken from intracranial EEG from five different epileptic patients. The EEG recordings were made as part of the diagnostics of the epileptic patients, prior and independently to this study. The EEG signals were either sampled at 512 or $1024 \mathrm{~Hz}$, depending on whether it was a more or less than 64 channel record.

Each signal was filtered by a band-pass fourth-order Butterworth filter, between 0.5 and $150 \mathrm{~Hz}$. Signals that were sampled at $1024 \mathrm{~Hz}$, were down-sampled to $512 \mathrm{~Hz}$.

Data were compiled into two different data sets: the " $F$ " set, are the data from the focal epileptic point, which was identified as the first electrode that measures the epileptic seizure. And the "N" set, are the data from the non-focal points. A non-focal point is any other point that didn't show the epileptic seizure.

Each data set was assembled by randomly selecting 7500 signals from a pool of 10240 samples, these samples were obtained by making windows of 20 seconds each. Recordings of seizure activity and three hours after were excluded from the data set. Before being included in the database, the signal was visually inspected to ensure non-significant artifacts were present. No clinical selection criterion, such as the presence or absence of epileptiform activity were applied.

\section{Theory}

Graph Theory has been used to study both the static and dynamic descriptions of complex systems. The "particles" or individual elements of the system are represented as nodes in the network and the interactions or links between these elements correspond to lines that join the nodes in pairs. The topology of these abstract objects allows to characterize certain types of network (small world, free scale, etc.) and associate them with some typical systems. The topology is determined by the number of links each node possesses (degree distribution), the clustering coefficient, or the average shortest path length between any pair of nodes. A comprehensive review of the subject can be found at http: //networksciencebook.com/[38].

\subsection{Feigenbaum graphs}

The Feigenbaum graphs are a tool that has been employed in the characterization time series data. By constructing a network from a given time series data set [39-41], the network structure extracts important information from said time series [42-46]. 

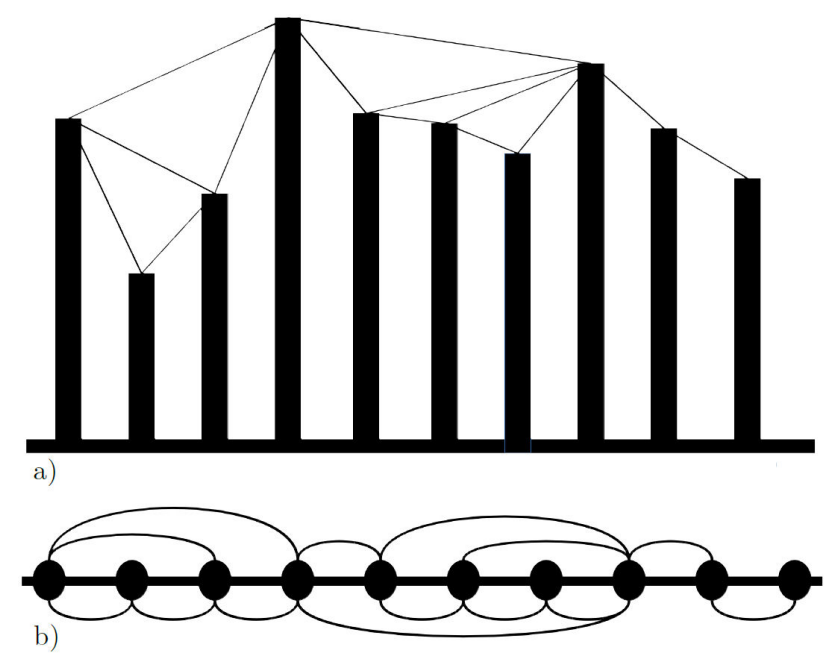

FIGURE 3. a) A data set where the lines indicate a link in the network. b) The network that results from the data in a), by following the mentioned procedure.

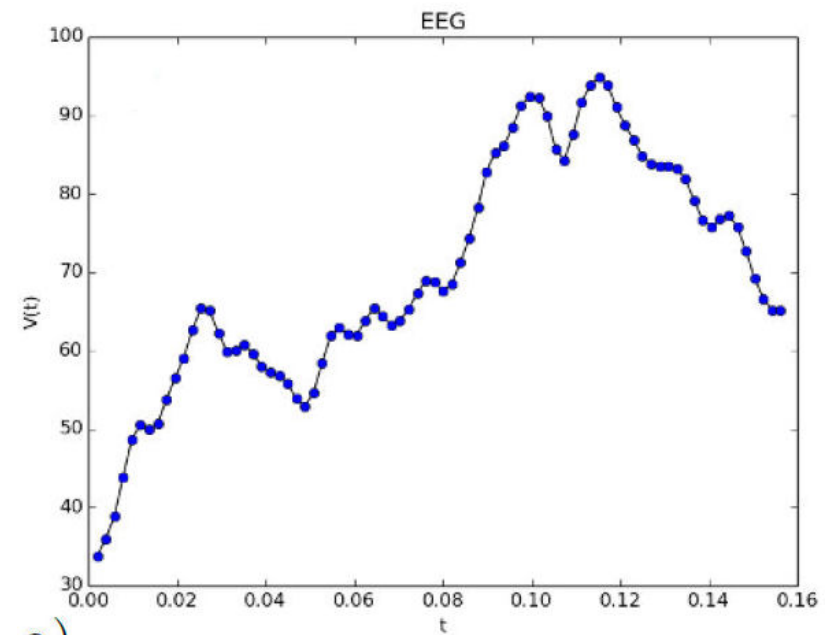

a)

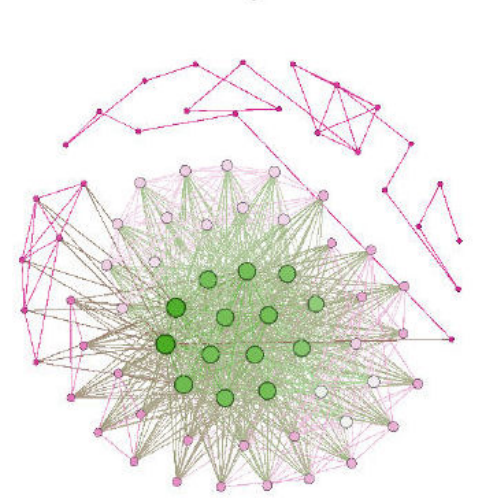

b)

FIGURE 4. a) EEG data from the [37] data set. b) The network that results from the data in a), by following the mentioned procedure.

To characterize the data sets and achieve a systematic method for identifying epileptic focal points, each signal from the corresponding data set, "N" or "F", was transformed into a Feigenbaum graph. Following the idea that most of the characteristics of the signals are translated to the topology of the network, the analysis of this topology is relevant.
The process for building the network is as follows: For each point $x_{i}$ in the data set, a node $i$ is added to the network. Then, for each pair of points $x_{i}$ and $x_{j}$ in the set, every time the criterion $x_{i}, x_{j}>x_{n}$ for all $n$, such that $i<n<j$ is met, an edge is added between nodes $i$ and $j[12,44]$.

Following the aforementioned procedure for the example data in Fig. 3a), the network from Fig. 3b) is built. By building the Feigenbaum network a new structure is met for the data, and so it can be analyzed as such.

Take the EEG from Fig. 4a). For each data point, a node is added to the network in Fig. 4b), and the edges are created following the procedure in Figs. 3a) and Fig. 3b).

\subsection{Statistical measurements}

Once the Feigenbaum graphs were built, an analysis of the structure of the graphs is in order. To find a measure to characterize them as "N" or " $\mathrm{F}$ " whichever was the case, some calculations on the topology of the networks are of use.

To this end, we calculated the average shortest path length for each graph, as it has a direct correlation to the size of the graph, and the data itself. On the other hand, we calculated the average clustering coefficient. It is a measurement of how the network is connected and correlates with how auto-similar the data are.

Getting the average clustering coefficient for each network, a single number is set to identify each of the time windows in the data sets. Also by calculating the average shortest path length for each network, a new single number is obtained to identify each EEG signal of 20 seconds.

The average clustering coefficient is calculated in the simple form (1) [47].

$$
C=\frac{1}{n} \sum_{v \in G} c_{v}
$$

where $n$ is the number of nodes in the network $G$, and $c_{v}$ is the clustering coefficient for each node $v$.

The clustering coefficient $c_{v}$ is calculated using Eq. (2) [49].

$$
c_{v}=\frac{2 T(v)}{k(v)(k(v)-1)}
$$

where $T(v)$ is the number of triangles through node $v$, and $k(v)$ is the degree of $v$.

So the average shortest path length is calculated employing the Eq. (3) [50].

$$
a=\sum_{s, t \in V} \frac{d(s, t)}{n(n-1)}
$$

Where $V$ is the set of nodes in the graph, $d(s, t)$ is the shortest path length from $s$ to $t$. And $n$ is the number of nodes in the graph.

Because the average shortest path length and the average clustering coefficient of each graph are calculated, each signal in each data set gets identified by two singular numbers. As these numbers on their own are not singularly defining, a 

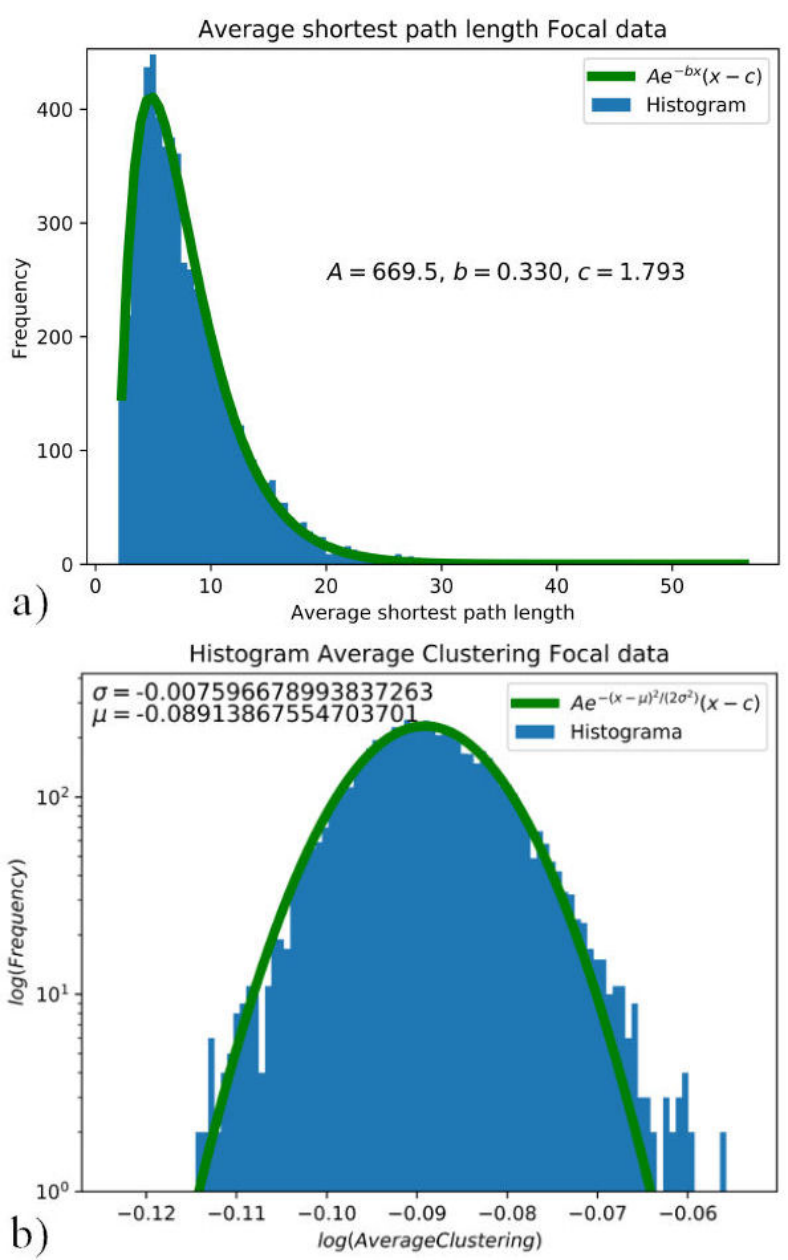

FIGURE 5. a) Distribution of the shortest path length for the focal data set, curve fitted to $A \exp (-b x)(x-c)$ where $A=669.5$, $b=0.330$ and $c=1.793$. b) Average clustering distribution for the focal data set, curve fitted to $A \exp \left(-(x-\mu)^{2} /\left(2 \sigma^{2}\right)\right)$, where $\sigma=-0.007$ and $\mu=-0.089$. Distributions of average shortest path length 5a) and average clustering coefficient $5 \mathrm{~b}$ ) for focal data.

statistical approach must be made. For this end, each individual set of parameters were assembled in distributions for said parameter and data set, as shown in Figs. 5a), 6a), 5a) and $6 b)$.

\section{Results and discussion}

As every signal is processed, the parameters for the average shortest path length, and average clustering coefficient, are calculated and placed on their respective distribution.

The distribution for the average clustering coefficient was assembled for each data set; hence, one distribution for the EEG signals in the focal set " $F$ ", and one for the EEG signal in the non-focal set "N". These distributions were curve fitted to identify the difference or lack of between data sets. For the average clustering coefficient, the histogram is presented in the $\log$ scale and fitted to $A \exp \left(-(x-\mu)^{2} /\left(2 \sigma^{2}\right)\right)$, as shown in Figs. 5b) and 6b). The tails on the average clus-
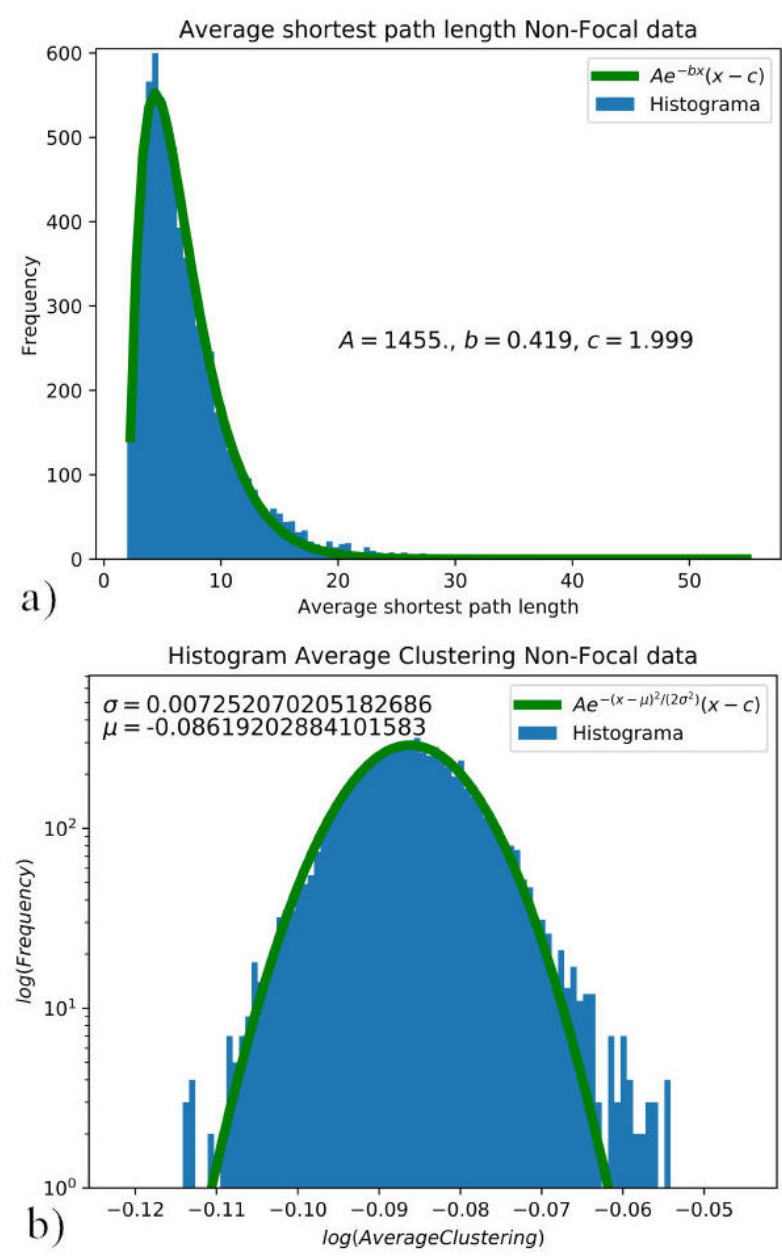

FIGURE 6. a) Distribution of the shortest path length for the nonfocal data set, curve fitted to $A \exp (-b x)(x-c)$ where $A=1455$, $b=0.419$ and $c=1.999$. b) Average clustering distribution for the non-focal data set, curve fitted to $A \exp \left(-(x-\mu)^{2} /\left(2 \sigma^{2}\right)\right)$, where $\sigma=-0.007$ and $\mu=-0.086$. Distributions of average shortest path length $6 a$ ), and average clustering coefficient $6 \mathrm{~b}$ ) for non-focal data.

tering coefficient distributions are not accounted for, without affecting the criteria for differentiation between focal and non-focal data. For the average shortest path length, the histogram is curve fitted to $A \exp (-b x)(x-c)$, as shown in Fig. 5a) and 6a).

In order to increase statistical accuracy and significance 20 subsets of 2000 signals were built from the original 7500 signal data set, for both " $\mathrm{N}$ " and " $\mathrm{F}$ " signals. These sets were assembled by the same means of randomly selecting the 2000 signals from the pool. For these new 20 sets of signals, the distributions of average clustering coefficient and average shortest path length were assembled and fitted, as shown in Figs. 7a) and 7b).

From the curve fitting of each new data subset, the relevant fitting parameters are established. The $b$ parameter from the $A \exp (-b x)(x-c)$ fit for the average shortest path length, is a way of characterizing both sets of data " $\mathrm{N}$ " and " $\mathrm{F}$ ". A comparison for this parameter is shown in Fig. 9a). The $\mu$ 

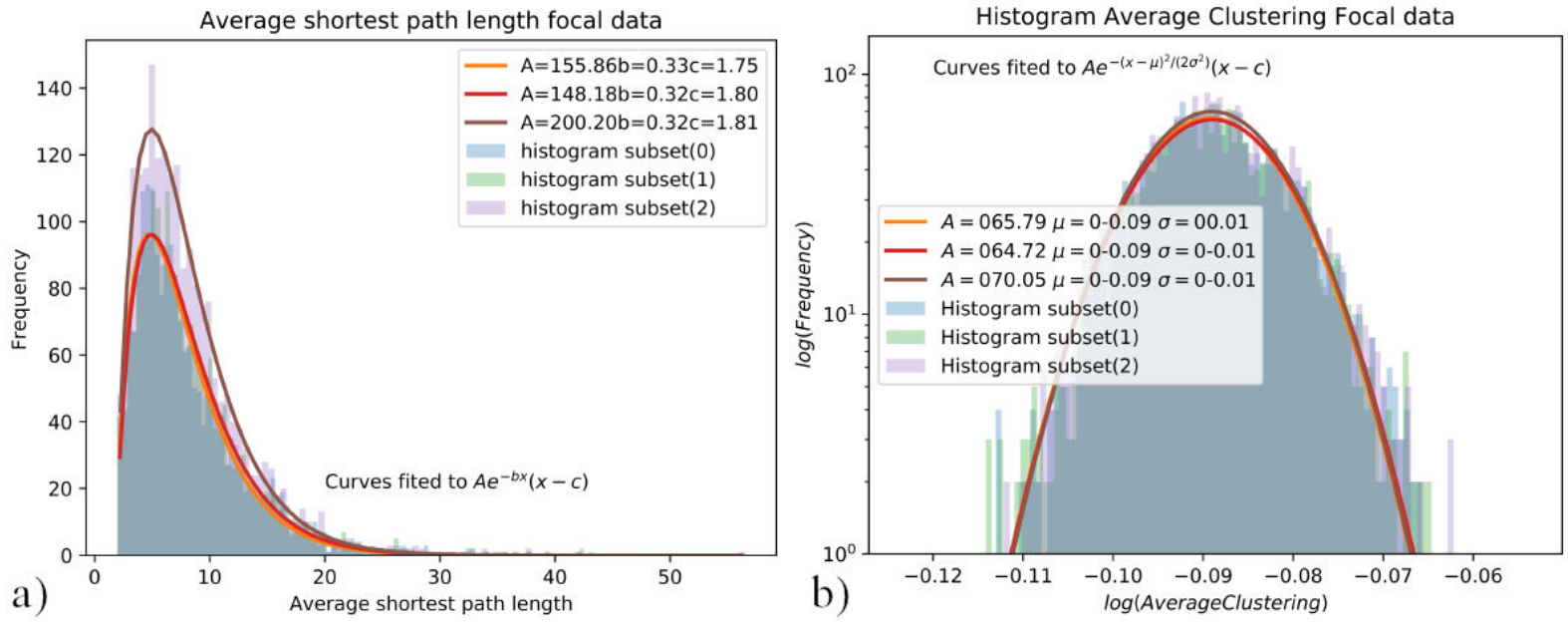

FIGURE 7. a) Distributions of the shortest path length, curve fitted to $A \exp (-b x)(x-c)$, b) Average clustering distributions, curve fitted to $A \exp \left(-(x-\mu)^{2} /\left(2 \sigma^{2}\right)\right)$. Distributions of average shortest path length $\left.7 \mathrm{a}\right)$, and average clustering coefficient $7 \mathrm{~b}$ ) for 3 subsets of 2000 samples of focal data.

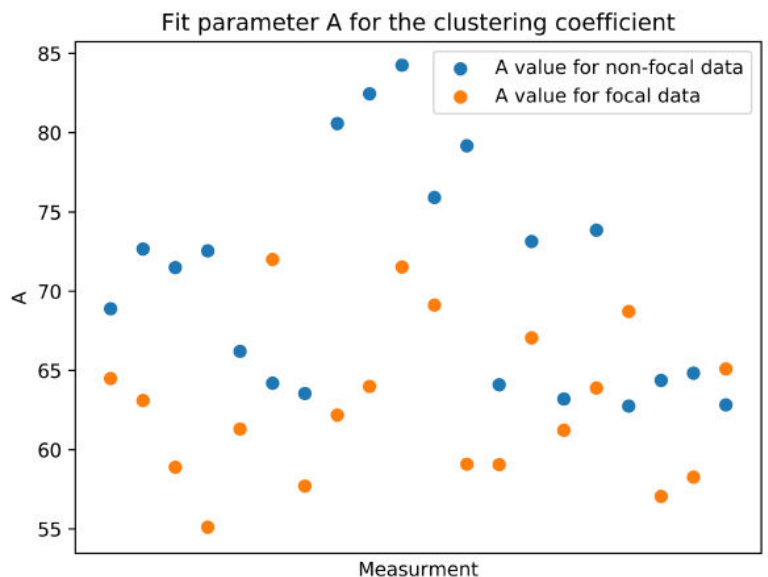

a) $A$

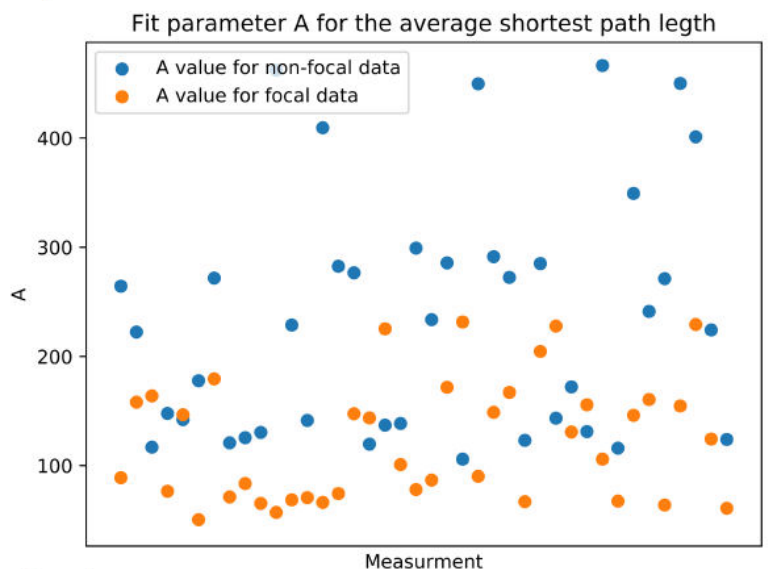

c) $A$

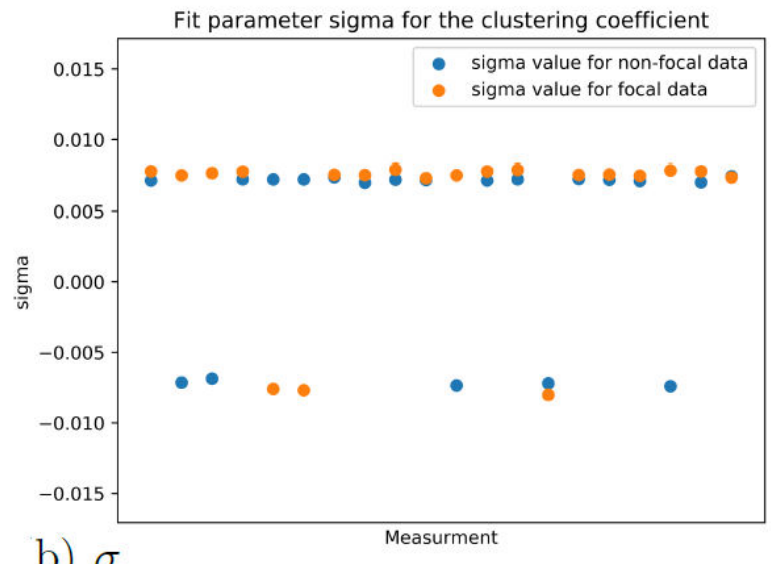

b) $\sigma$

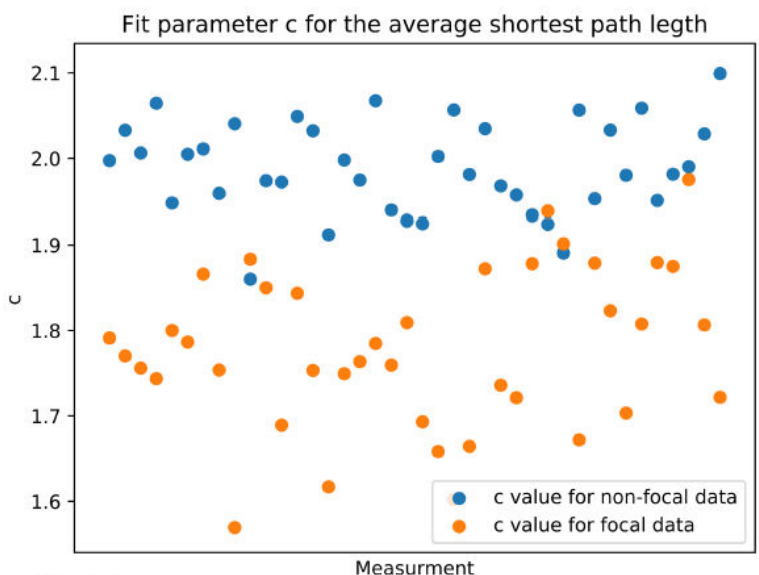

d) $C$

FIGURE 8. Fitting parameters for the average clustering coefficient and average shortest path length distributions.

parameter from the $A \exp \left(-(x-\mu)^{2} /\left(2 \sigma^{2}\right)\right)$ fit for the average clustering coefficient is also a way of differentiating between both data sets "N" and "F" as shown in Fig. 9b).
Parameters $b$ and $\mu$ are chosen since they have the biggest weight in each curve fitting. As the data is assumed to have different structures for the focal "F" and non-focal "N" data, 

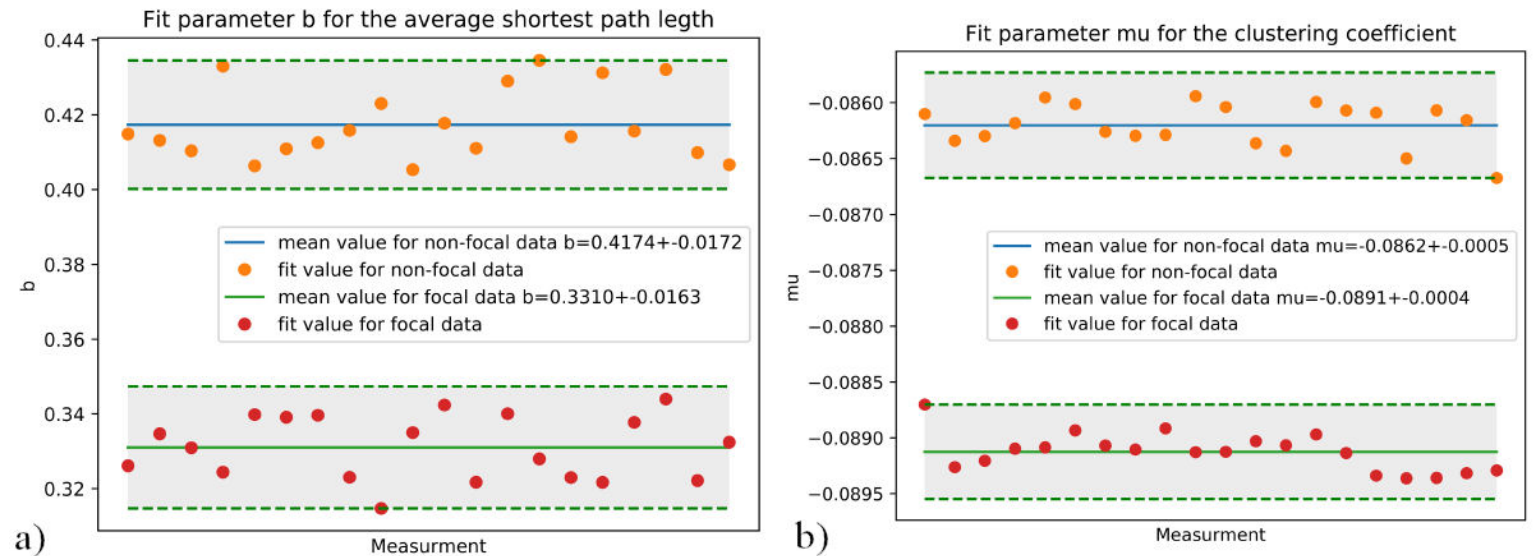

FIgURE 9.a) Comparison of the value of the $b$ parameter for "N" and "F" data. b) Comparison of the $\mu$ parameter for "N" and "F" data. Relevant fitting parameters for Average clustering coefficient 9a) and average shortest path length 9b), 20 distributions of 2000 samples.

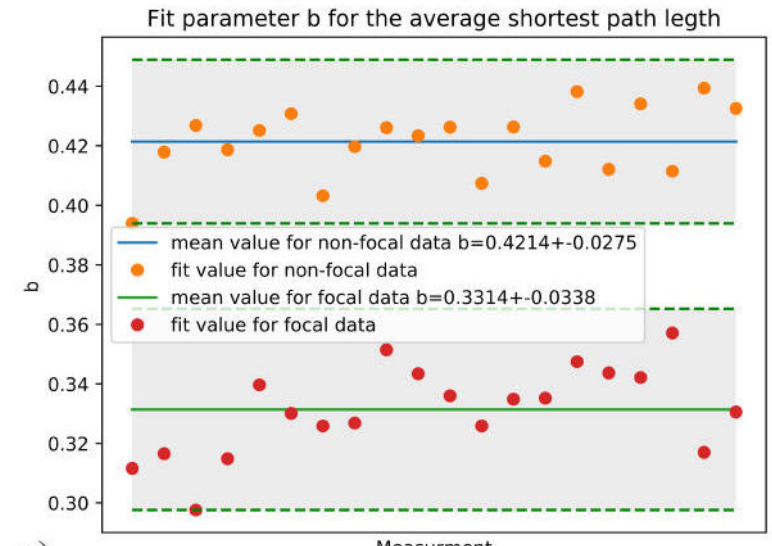

a)

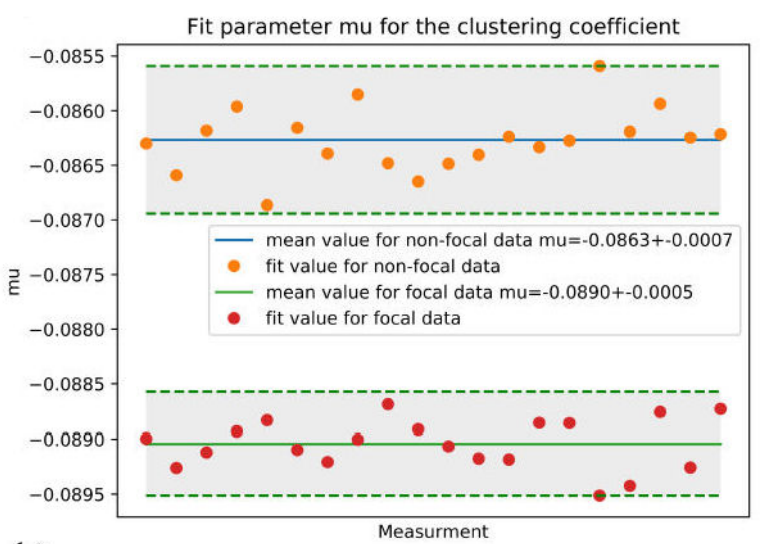

b)

FIGURE 10. a) Comparison of the $b$ parameter for "N" and "F" data. b) Comparison of the $\mu$ parameter for "N" and "F" data. Relevant fitting parameters for Average clustering coefficient 10a) and average shortest path length 10b), 20 distributions of 1000 samples.

TABLE I. Values of $b$ and $\mu$ for focal and non-focal data.

\begin{tabular}{ccc}
\hline & $\mu$ & $b$ \\
\hline Focal data & $-0.0891 \pm 0.0004$ & $0.3310 \pm 0.0163$ \\
2000 samples & & \\
Non-focal data & $-0.0862 \pm 0.0005$ & $0.4174 \pm 0.0172$ \\
2000 samples & & \\
Focal data & $-0.0890 \pm 0.0005$ & $0.3314 \pm 0.0338$ \\
1000 samples & & \\
Non-focal data & $-0.0863 \pm 0.0007$ & $0.4214 \pm 0.0275$ \\
1000 samples & & \\
\hline
\end{tabular}

these parameters are the most important. Furthermore, the other parameters are non-significant to make any decisions if the data comes from focal or non-focal EEG, as shown in the plots for the $\sigma, A$, and $c$ parameters in Fig. 8 .

Following the same process, another 20 subsets of 1000 signals were built, for both "N" and "F" signals. The calcu- lations of the average shortest path length and average clustering coefficient for these subsets were also assembled in distributions and fitted. The comparison of relevant fitting parameters, $b$ for the average shortest path length in Fig. 10a) and $\mu$ for the clustering coefficient in Fig. 10b) are shown.

In Table I, the values to differentiate between focal and non-focal data are presented. For the curve fitting of the average clustering coefficient $A \exp (-b x)(x-c)$ the parameter $b$. And the parameter $\mu$ for the curve fit $A \exp (-(x-$ $\left.\mu)^{2} /\left(2 \sigma^{2}\right)\right)$ of the average shortest path length. The differences shown in Table I are consistent when calculating for the entire data of 75000 samples, where $b=0.419$ for nonfocal data and $b=0.330$ for focal data, and $\mu=-0.089$ for focal data and $\mu=0.086$ for non-focal data.

As a way to measure the effectiveness of these results, complementary measurements were made. The Hurst exponent, sample entropy approximate entropy and fractal dimension of the EEG recordings were calculated. Distributions for each of these measurements are shown in Fig. 11, for both focal and non-focal EEG data. 


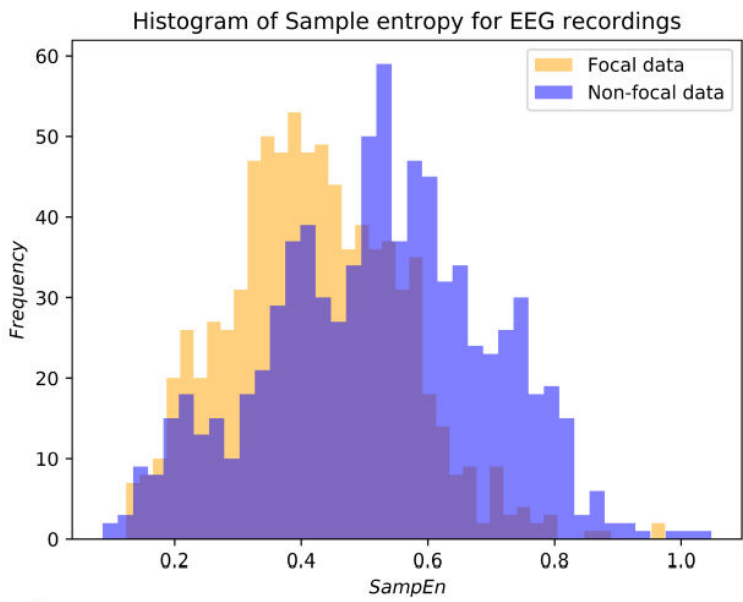

a)

Sample entropy

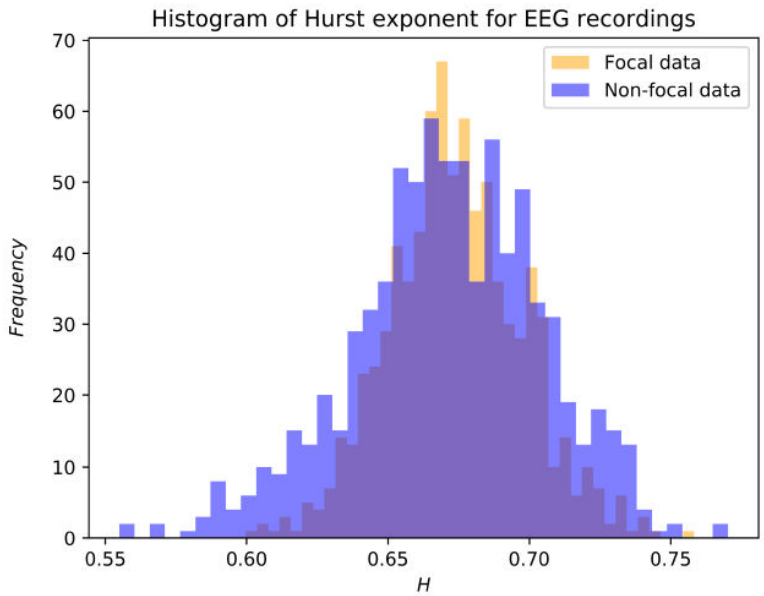

c)

Hurst exponent

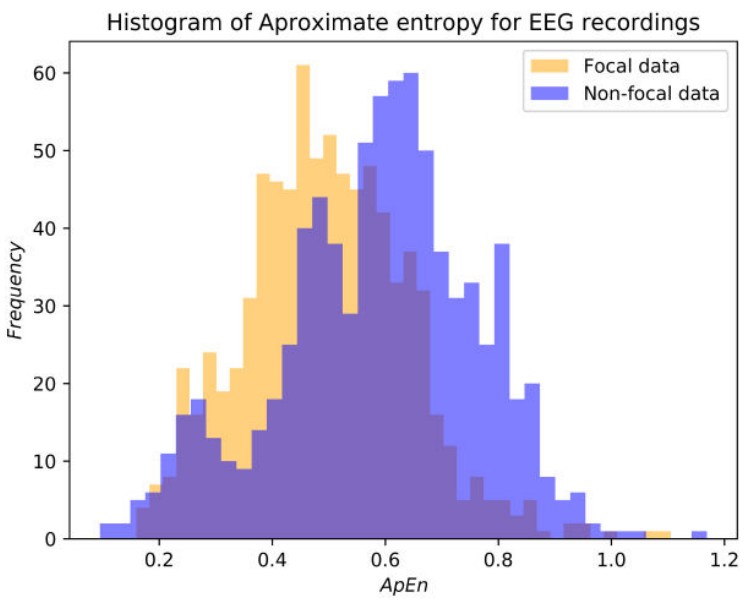

b)

Approximate entropy

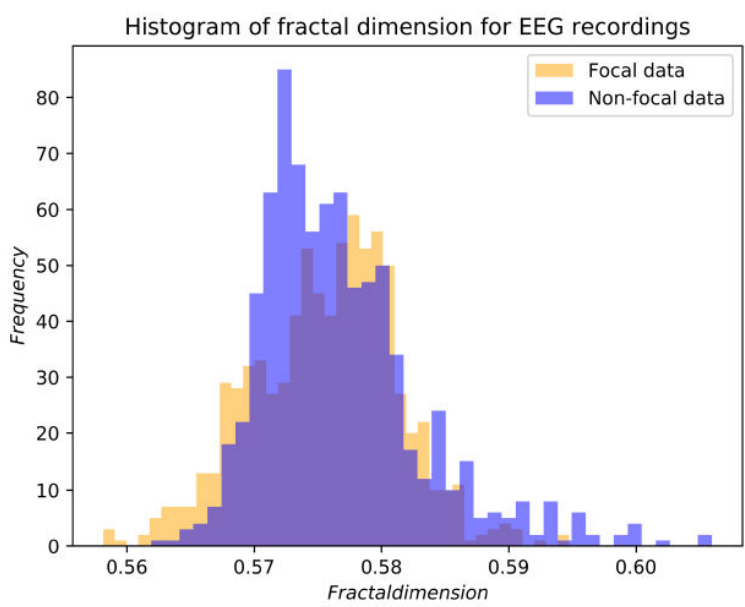

d)

FIGURE 11. Non-linear measurements for EEG recordings.

TABLE II. KS-test results for different measurements

\begin{tabular}{ccc}
\hline & ks statistic & P value \\
\hline Fit parameter $\mathrm{b}$ for $\mathrm{spl}$ & 1.0 & 0 \\
Fit parameter $\mu$ for $\mathrm{C}$ & 1.0 & 0 \\
\hline
\end{tabular}

For 2000

sample data sets

\begin{tabular}{ccc}
\hline Fit parameter $\mathrm{b}$ for $\mathrm{spl}$ & 1.0 & $1.4508 e^{-11}$ \\
Fit parameter $\mu$ for $\mathrm{C}$ & 1.0 & $1.4508 e^{-11}$ \\
\hline
\end{tabular}

For 1000

sample data sets

\begin{tabular}{ccc}
\hline Fit parameter $\mathrm{b}$ for spl & 1.0 & $1.4508 e^{-11}$ \\
Fit parameter $\mu$ for $\mathrm{C}$ & 1.0 & $1.4508 e^{-11}$ \\
\hline Sample entropy & 0.30597 & $1.26894 e^{-33}$ \\
Approximate entropy & 0.28731 & $1.21786 e^{-29}$ \\
Hurst exponent & 0.10199 & $4.62265 e^{-4}$ \\
Fractal dimension & 0.09577 & $1.24584 e^{-3}$ \\
\hline
\end{tabular}

The usefulness of these measurements to differentiate each individual set of EEG recordings is estimated by means of the Kolmogorov-Smirnov (KS) statistic test. The results for the KS tests are presented in Table II, where one can see the statistical significance of each property of the EEG.

\section{Conclusion}

In this work, a new method is proposed to identify epileptic focal zones from the Andrzejak et al. database [37]. It is managed by assembling Feigenbaum graphs and calculating distributions for the average shortest path length and average clustering coefficient from every data set. Fitting the average clustering coefficient to $A \exp \left(-(x-\mu)^{2} /\left(2 \sigma^{2}\right)\right)$ and observing the $\mu$ parameter, if $\mu=-0.089 \pm 0.0005$ the data is said to be focal, also if $\mu=-0.0863 \pm 0.0007$ the data is said to be non-focal. This yields a differentiating factor between both focal and non-focal signals.

For the average shortest path length, the distribution, curve fitting to $A \exp (-b x)(x-c)$ and checking for the $b$ parameter. If $b=0.4214 \pm 0.0275$, the data are said to be 
non-focal, and if $b=0.3314 \pm 0.0338$, the data are said to be focal, giving another criterion to differentiate between focal and non-focal signals.

As shown in Table I, this new approach yields better confidence to differentiate between focal and non-focal EEG. For the KS test, the $b$ parameter and $\mu$ parameter can be used with a p-value of 0 and $\mathrm{KS}$ - statistic value of 1 , hence is a much better differentiating factor than either of the Approximate entropy, Sample entropy, Hurst exponent, or fractal dimension factors.

Following the idea and how the data set are assembled, this measurement could be calculated for a single patient by assembling a data set created from segments -time windowsof EEG studies no matter the timeline, and analyzing each signal. The calculation of the curve fit for the average shortest path length distribution, and the average clustering coefficient of the Feigenbaum graphs of the data set can be used to identify the focal EEG electrode readings.

Because the results are less scattered for the 2000 sample set than the 1000 sample set, it suggests that 2000 samples should be used for better results, although 1000 samples can be used at a lesser computational cost, since the KS-test values are the same for both sample sizes, $K S-$ statistic $=1$, and $p-$ value $=1.4508 e^{-11}$. This could help the physician assess a better diagnosis for the patient in the determination of epilepsy focal sites.

Subject to considering other databases and other probably more complex cases, we see that, with this technique, it is possible to distinguish between the signal that comes from an epileptic focus and another that does not, which can be of great value both in diagnosis and in the practical determination of focal sites for surgical intervention.

\section{Acknowledgments}

We are grateful for the facilities provided by the Laboratorio Nacional de Supercómputo (LNS) del Sureste de México to obtain these results.
1. Epilepsy Foundation. https://www.epilepsy.com/ Accessed: 2018- 08-27.

2. Deborah Buck, Gus A Baker, Ann Jacoby, David F Smith, and David W Chadwick. Patients' Experiences of Injury as a Result of Epilepsy. Epilepsia, 38 (1997) 439. https : // doi .org/ $10.1111 / j .1528-1157.1997 . t b 01733 . x$

3. Leone Ridsdale. Avoiding premature death in epilepsy. $B M J$, 350 (2015). https: //doi.org/10.1136/bmj.h718

4. A. T. Berg, F. A. Zelko, S. R. Levy, and F. M. Testa. Age at onset of epilepsy, pharmacoresistance, and cognitive outcomes: a prospective cohort study. Neurology, 79 (2012) 1384. https : //doi.org/10.1212/WNL.0b013e31826c1b55

5. W. Allen Hauser, John F. Annegers, and Leonard T. Kurland. Incidence of Epilepsy and Unprovoked Seizures in Rochester, Minnesota: 1935- 1984. Epilepsia, 34 (1935) 453. https://doi.org/10.1111/j.1528-1157.1993. tb02586.x

6. W. Ian McDonald et al., Wolinsky. Recommended diagnostic criteria for multiple sclerosis: Guidelines from the international panel on the diagnosis of multiple sclerosis. Annals of Neurology, $\mathbf{5 0}$ 121.https://doi.org/10.1002/ana.1032

7. Srimonti Dutta, Dipak Ghosh, Shukla Samanta, and Santanu Dey. Multifractal parameters as an indication of different physiological and pathological states of the human brain. Physica A: Statistical Mechanics and its Applications, 396 (2014) 155. https://doi.org/10.1016/j. physa.2013.11.014

8. Klaus Lehnertz, Gerrit Ansmann, Stephan Bialonski, Henning Dickten, Christian Geier, and Stephan Porz. Evolving networks in the human epileptic brain. Physica D: Nonlinear Phenomena, 267 (2014) 715. Evolving Dynamical Networks. https : //doi.org/10.1016/j.physd.2013.06.009
9. Klaus Lehnertz, Stephan Bialonski, Marie-Therese Horstmann, Dieter Krug, Alexander Rothkegel, Matthus Staniek, and Tobias Wagner. Synchronization phenomena in human epileptic brain networks. Journal of Neuroscience Methods, 183 (2009) 4248. BrainModes: A Principled Approach to Modeling and Measuring Large-Scale Neuronal Activity. https://doi. org/10.1016/j.jneumeth.2009.05.015

10. Mikail Rubinov and Olaf Sporns. Complex network measures of brain connectivity: Uses and interpretations. Neurolmage, 52 (2010) 10591069 . Computational Models of the Brain. https://doi.org/10.1016/j.neuroimage. 2009.10 .003

11. Olaf Sporns and Richard F. Betzel. Modular brain networks. Annual Review of Psychology, 67 (2016) 613640. PMID: 26393868. https://doi.org/10.1146/ annurev-psych-122414-033634

12. N. M. G Bodde, J. L. Brooks, G. A. Baker, P. A. J. M. Boon, J. G. M. Hendriksen, O. G. Mulder, and A. P. Aldenkamp, Psychogenic non-epileptic seizures-definition, etiology, treatment and prognostic issues: a critical review. Seizure, 18 (2009) 543. https: / doi .org/10.1016/j. seizure.2009.06.006

13. M. Bandarabadi, J. Rasekhi, C. A. Teixeira, M. R. Karami, and A. Dourado. On the proper selection of preictal period for seizure prediction. Epilepsy Behav, 46 (2015) 158. https: //doi.org/10.1016/j.yebeh.2015.03.01

14. L. Kuhlmann et al., Epilepsyecosystem.org: crowd-sourcing reproducible seizure prediction with long-term human intracranial EEG. Brain, (2018). https://doi.org/10.1093/ brain/awy210

15. M. R. Nuwer. The development of EEG brain mapping. J Clin Neuro- physiol, 7 (1990) 459. 10.1097/ 00004691-199010000-00002 
16. Dipak Ghosh, Srimonti Dutta, and Sayantan Chakraborty. Multifractal detrended cross-correlation analysis for epileptic patient in seizure and seizure free status. Chaos, Solitons \& Fractals, 67 (2014) 1. https://doi.org/10.1016/j. chaos.2014.06.010

17. R.H. Shumway and D.S. Stoffer. Time Series Analysis and Its Applications: With R Examples. Springer Texts in Statistics. Springer International Publishing, 2017).

18. Siuly Siuly, Yan Li, and Yanchun Zhang. EEG signal analysis and classification: techniques and applications. (Springer, 2019).

19. Oliver Faust, U. Rajendra Acharya, Hojjat Adeli, and Amir Adeli. Wavelet-based EEG processing for computer-aided seizure detection and epilepsy diagnosis. Seizure, 26 (2015) 56. https://doi.org/10.1016/j.seizure.2015. 01.012

20. Sheng-Fu Liang, Hsu-Chuan Wang, and Wan-Lin Chang. Combination of EEG Complexity and Spectral Analysis for Epilepsy Diagnosis and Seizure Detection. EURASIP J. Adv. Signal Process, 2010, (2010) 62:1-62:15. https://doi. org/10.1155/2010/853434

21. U. Rajendra Acharya, S. Vinitha Sree, G. Swapna, Roshan Joy Martis, and Jasjit S. Suri. Automated EEG analysis of epilepsy: A review. Knowledge-Based Systems, 45 (2013) 147. https: //doi.org/10.1016/j.knosys.2013.02.014

22. Mehran Ahmadlou, Anahita Adeli, Ricardo Bajo, and Hojjat Adeli. Complexity of functional connectivity networks in mild cognitive impairment subjects during a working memory task. Clinical Neurophysiology, 125 (2014) 694. https : //doi.org/10.1016/j.clinph.2013.08.033

23. U. R. Acharya, V. K. Sudarshan, H. Adeli, J. Santhosh, J. E. Koh, and A. Adeli. Computer-Aided Diagnosis of Depression Using EEG Signals. Eur. Neurol 73 (2015) 329. https : //doi.org/10.1159/000381950

24. R.O. Duda, P.E. Hart, and D.G. Stork, Pattern Classification, New York: John Wiley \& Sons, 2001, pp. xx 654, ISBN: 0471-05669-3. J. ClassiFication, 24 (2007) 305. $10.1007 /$ S00357-007-0015-9

25. P Welch. The use of fast Fourier transform for the estimation of power spectra: A method based on time averaging over short, modiFied periodograms. IEEE Trans. Audio Electroacoust 15 (1967) 70. DOI : 10.1109/TAU.1967.1161901

26. A. Scheffler, D. Telesca, Q. Li, C. A. Sugar, C. Distefano, S. Jeste, and D. Senturk. Hybrid principal components analysis for region-referenced longitudinal functional EEG data. Biostatistics, (2018).

27. H. Adeli, Z. Zhou, and N. Dadmehr. Analysis of EEG records in an epileptic patient using wavelet transform. J. Neurosci. Methods, 123 (2003) 69. https://doi.org/10.1016/ S0165-0270(02)00340-0

28. S. Ghosh-Dastidar, H. Adeli, and N. Dadmehr. Mixed-Band Wavelet- Chaos-Neural Network Methodology for Epilepsy and Epileptic Seizure Detection. IEEE Transactions on Biomedical Engineering, 54(9):1545 (2007). DOI : 10 . 1109/ TBME.2007.891945

29. Z. Czechowski, M. Lovallo, and L. Telesca. Multifractal analysis of visibility graph-based Ito-related connectivity time series.
Chaos, 26 (2016) 023118. https://doi.org/10.1063/ 1.4942582

30. U Rajendra Acharya, Hamido Fujita, Vidya K Sudarshan, Shreya Bhat, and Joel En Wei Koh. Application of entropies for automated diagnosis of epilepsy using EEG signals: A review. 88 (2015) 08.

31. G. Ouyang, J. Li, X. Liu, and X. Li. Dynamic characteristics of absence EEG recordings with multiscale permutation entropy analysis. Epilepsy Res., 104 (2013) 246. https : // doi.org/10.1016/j.eplepsyres.2012.11.003

32. M Vetterli and C Herley. Wavelets and Filter banks: theory and design. IEEE Trans. Signal Process., 40 (1992) 2207. $10.1109 / 78.157221$

33. Juan P. Amezquita-Sanchez and Hojjat Adeli. A New Musicempirical Wavelet Transform Methodology for Time-frequency Analysis of Noisy Nonlinear and Non-stationary Signals. Digit. Signal Process., 45 (2015) 55. https://doi.org/10. $1016 / j . d s p .2015 .06 .013$

34. Zhong-Ke Gao, Qing Cai, Yu-Xuan Yang, Na Dong, and Shan-Shan Zhang. Visibility graph from adaptive optimal kernel time-frequency representation for classiFication of epileptiform eeg. International Jour- nal of Neural Systems, 27 (2017) 1750005. PMID: 27832712. https://doi.org/ $10.1142 / \mathrm{S} 0129065717500058$

35. Salim Lahmiri. Generalized hurst exponent estimates differentiate eeg signals of healthy and epileptic patients. Physica A: Statistical Mechanics and its Applications, 490 (2018) 378385. https://doi.org/10.1016/j. physa.2017.08.084

36. Lei Wang, Xi Long, Johan B.A.M. Arends, and Ronald M. Aarts. Eeg analysis of seizure patterns using visibility graphs for detection of generalized seizures. Journal of Neuroscience Methods, 290 (2017) 8594. https : //doi.org/ $10.1016 / j \cdot j n e u m e t h .2017 .07 .013$

37. Ralph G Andrzejak, Kaspar Schindler, and Christian Rummel. Nonrandomness, nonlinear dependence, and nonstationarity of electroencephalographic recordings from epilepsy patients. Phys. Rev. E Stat. Nonlin. Soft Matter Phys., 86 (2012) 046206. https://doi.org/10.1103/PhysRevE.86. 046206

38. Network science by albert-lázló barabási. http: //networksciencebook.com/ Accessed: 2019-0930

39. Zhong-Ke Gao, Yu-Xuan Yang, Peng-Cheng Fang, Yong Zou, Cheng-Yi Xia, and Meng Du. Multiscale complex network for analyzing experimental multivariate time series. 109 (2015) 02. https://doi.org/10.1209/0295-5075/ $109 / 30005$

40. S. Bhaduri and D. Ghosh. Electroencephalographic Data Analysis With Visibility Graph Technique for Quantitative Assessment of Brain Dysfunction. Clin EEG Neurosci, 46 (2015) 218. https://doi.org/10.1177/1550059414526186

41. A. Olamat, P. Shams, and A. Akan. Synchronization analysis of eeg epilepsy by visibility graph similarity. In 2018 Medical Technologies National Congress (TIPTEKNO), pages 1-4, Nov (2018). DOI: $10.1109 /$ TIPTEKNO.2018.8596770 
42. Xiaoke Xu, Jie Zhang, and Michael Small. Superfamily phenomena and motifs of networks induced from time series. Proc. Natl. Acad. Sci. U. S. A., 105 (2008) 19601. https : / / doi. org/10.1073/pnas.0806082105

43. Lucas Lacasa and Raul Toral. Description of stochastic and chaotic series using visibility graphs. Phys. Rev. E Stat. Nonlin. Soft Matter Phys., 82 (2010) 036120. https://doi.org/ $10.1103 /$ PhysRevE.82.036120

44. Bartolo Luque, Lucas Lacasa, Fernando J Ballesteros, and Alberto Robledo. Feigenbaum graphs: a complex network perspective of chaos. PLoS One, 6 (2011) e22411. https: // doi.org/10.1371/journal.pone.0022411

45. Zhong-Ke Gao and Ning-De Jin. A directed weighted complex network for characterizing chaotic dynamics from time series. Nonlinear Analysis: Real World Applications, 13 (2012) 947. https://doi.org/10.1016/j.nonrwa.2011. 08.029

46. H. Xu, J. Dai, J. Li, J. Wang, and F. Hou. Research of eeg signal based on permutation entropy and limited penetrable visibility graph. In 2018 11th International Congress on Im- age and Signal Processing, BioMedical Engineering and Informatics (CISP-BMEI), pages 1-5, Oct (2018). DOI10.1109/ CISP-BMEI.2018.8633121

47. Jari Saramäki, Mikko Kivelä, Jukka-Pekka Onnela, Kimmo Kaski, and János Kertész. Generalizations of the clustering coefficient to weighted complex networks. Phys. Rev. E, 75 (2007) 027105. DOI10.1109/CISP-BMEI.2018.8633121

48. Marcus Kaiser. Mean clustering coefficients: the role of isolated nodes and leafs on clustering measures for small-world networks. New Journal of Physics, 10 (2008) 083042. https : //doi.org/10.1088/1367-2630/10/8/083042

49. Jukka-Pekka Onnela, Jari Saramäki, János Kertész, and Kimmo Kaski. Intensity and coherence of motifs in weighted complex networks. Phys. Rev. E, 71 (2005) 065103. https://doi. org/10.1103/PhysRevE.71.065103

50. Danny Z. Chen. Developing Algorithms and Software for Geometric Path Planning Problems. ACM Comput. Surv., 28(4es) (1996). https://doi.org/10.1145/242224. 242246 